\title{
COVID-19 Infection Presenting as Paroxysmal Nocturnal Hemoglobinuria
}

\author{
Adam Hines $^{1}$, Nausheen Hakim ${ }^{1}$, and Jacqueline Barrientos ${ }^{2}$ \\ ${ }^{1}$ Long Island Jewish Medical Center Northwell Health Cancer Institute \\ ${ }^{2}$ Hofstra Northwell School of Medicine
}

March 5, 2021

\begin{abstract}
We present the case of a 35-year-old male with no medical history who presented with pancytopenia and melena and was found to have PNH in the setting of acute COVID-19 infection. Other infectious, autoimmune, and malignant etiologies were ruled out. It is unclear if this was an acquired PIG-A mutation.
\end{abstract}

COVID-19 Infection Presenting as Paroxysmal Nocturnal Hemoglobinuria

Authors: Adam Hines MD ${ }^{1}$, Nausheen Hakim DO ${ }^{1}$, Jacqueline Barrientos MD, MS ${ }^{1}$

Northwell Health Cancer Institute ${ }^{1}$

Corresponding Author:

Jacqueline Barrientos MD, MS

450 Lakeville Road

North New Hyde Park, NY

Jbarrientos@northwell.edu

Running Title: COVID-19 Presenting as PNH

Keywords: COVID-19, SARS-CoV2, PNH

Word Count: 772

Figures: 3

There was no funding provided for this manuscript.

There are no conflict of interests to disclose for the authors stated above.

The patient consented to the publication of images and/or information in regards to this case.

Abstract:

We present the case of a 35-year-old male with no past medical history who presented with pancytopenia and melena and was found to have PNH in the setting of acute COVID-19 infection. Other infectious, autoimmune, and malignant etiologies were ruled out. It is unclear if this was an acquired PIG-A mutation or an expansion of a preexisting mutation in the setting of COVID-19 infection. Emerging evidence shows that the SARS-CoV-2 spike protein directly activates complement by engaging the alternate complement 
pathway which could indicate this is an acquired PIG-A expanded mutation. To our knowledge this is the first case of COVID-19 infection presenting as PNH.

A 35-year-old African-American man with history of migraines presented to the emergency room with headaches and melena associated with fatigue and inappetence for two weeks. He was initially admitted to the intensive care unit for suspected gastrointestinal bleed given severe pancytopenia. He denied any fevers, chills, cough, myalgias, dyspnea, difficulty breathing, diarrhea, anosmia, or ageusia. He was transfused two units of packed red blood cells and transferred to general medical floor as he was hemodynamically stable. At home he was taking ibuprofen $800 \mathrm{mg}$ daily for one week due to his headaches. Physical exam was unremarkable.

Initial laboratory tests showed white blood cell count $(1.70 \mathrm{~K} / \mathrm{uL})$, absolute neutrophil count $(0.82 \mathrm{~K} / \mathrm{uL})$, absolute lymphocyte count $(0.65 \mathrm{~K} / \mathrm{uL})$, hemoglobin $(5.0 \mathrm{~g} / \mathrm{dL})$, platelet count $(12 \mathrm{~K} / \mathrm{uL})$, with normal prothrombin time, partial thromboplastin time, international normalized ratio, D-dimer, fibrinogen, and normal renal and hepatic function. Computed Tomography of chest/abdomen/pelvis revealed bilateral lower lobe opacities.

COVID-19 by nasopharyngeal polymerase chain reaction (PCR) was positive on Day 1. Viral studies, including parvovirus, human immunodeficiency virus, cytomegalovirus, Epstein-Barr, varicella zoster, hepatitis B and C, were negative. Iron studies, vitamin B12, folate, Coombs, and urinalysis were normal. Exhaustive Rheumatological testing was unremarkable including complement 3 / 4/ 5 levels, Aldolase, cyclic citrullinated peptide antibody, anti-nuclear factor antibody, anti-smith antibody, anti-RNP antibody, antidouble stranded DNA antibody, anti-SSA and anti-SSB antibody, scleroderma and centromere antibodies. Abnormal laboratory studies included undetectable haptoglobin $(<20 \mathrm{mg} / \mathrm{dL})$, with elevated lactate dehydrogenase $(794 \mathrm{U} / \mathrm{L})$, ferritin $(503 \mathrm{ng} / \mathrm{dL})$, and erythrocyte sedimentation rate $(38 \mathrm{~mm} / \mathrm{hr})$. Blood smear showed poikilocytosis and large granular lymphocytes (LGL) [Figure 1]. Peripheral T-Cell receptor (TCR) gene rearrangement showed clonality in TCR $\beta$ and TCR $\gamma$ [Figure 2]. Peripheral flow cytometry revealed normal myeloid and lymphocyte immunophenotype with increased natural killer-like T-cells (20\%). Serum protein electrophoresis was normal with increased serum kappa/lambda free light chain ratio (2.09; normal 0.26-1.65), normal by day 17. Serum and urine immunofixation showed no monoclonality. Bone marrow biopsy showed trilineage hematopoiesis with maturation. Glycosylphosphatidylinositol-linked antigen testing revealed "classical Paroxysmal Nocturnal Hemoglobinuria (PNH)" showing RBC-Complete Antigen (Ag) Loss 4.34\% (Reference Range (RR) 0.00-0.01), Granulocytes 42.55\% (RR 0.00-0.01), Monocytes 45.84\% (RR 0.00-0.05) [Figure 3]. Due to his persistent headaches a Magnetic Resonance Imaging (MRI) of the brain was performed which showed nonspecific patchy elliptically shaped areas of restricted diffusion and low marrow signal within the left parietal calvarium, cervical vertebral bodies, posterior elements, and clivus with minimal if any associated enhancement. The patient empirically received high-dose pulse steroid and intravenous immunoglobulin without improvement. He was discharged home to start eculizumab as outpatient.

Here we present the case of a patient with new onset PNH in the setting of acute COVID-19 infection without any of the typical symptoms associated with the disease caused by severe acute respiratory syndrome coronavirus 2 (SARS-CoV-2). PCR testing was positive on days 1 and 7 of hospitalization, and subsequently tested negative on days 13 and 14. COVID IgG and IgA antibodies were negative on days 2 and day 4, confirming this was an acute infection. To our knowledge, this is the first report of a case of COVID-19 presenting with isolated pancytopenia from PNH without any of the commonly associated symptoms from the acute infection. Review of the literature shows the report of a single case of a patient with a known history of PNH developing acute flare possibly precipitated by COVID-19 infection. ${ }^{1}$

There is a known association with LGL clonal expansion in PNH, though unlikely relevant unless persistently cytopenic post PNH therapy. ${ }^{2}$ There was an increase in NK-like T cells, CD $57+/ \mathrm{CD} 3+$ comprising $10.3 \%$ of the total population with different subtypes such as CD57+/CD8+ comprising $5.9 \%$ of the total population. An increase in these populations can be seen in conditions such inflammatory disease, viral infections, following treatment with chemotherapy or transplant patients and in large granular lymphocyte leukemia. T-cell rearrangement studies showed discrete bands in both TCR-beta and TCR-gamma analysis, suggesting 
a clonal T-cell population. However, the clinical significance of these findings is unclear in the setting of pancytopenia and the acute COVID-19 infection.

$\mathrm{PNH}$ is an X-linked acquired somatic mutation of the phosphatidylinositol glycan class A (PIG-A) gene that can cause bone marrow failure, complement mediated hemolysis, and thrombophilia. ${ }^{5}$ This mutation causes impaired expression of CD55 and CD59 (complement regulators) which causes dysregulated complement activation. ${ }^{5}$

$\mathrm{Yu}$ et al demonstrated that SARS-CoV-2 spike proteins activate complement by engaging the alternative complement pathway. ${ }^{3}$ This activation can cause complement mediated damage such as endothelial injury, hemolysis, and contribute to end organ damage. ${ }^{4}$ Prior to this admission, the patient had not sought medical care in years, thus prior laboratory data for the patient is not available. It is possible that he had subclinical PNH that was discovered only during the acute COVID-19 infection when he became symptomatic. It is unclear if the acute COVID-19 infection caused an acquired PIG-A mutation or expanded an existing PIG-A mutation.

\section{References}

1. Schüller, H., Klein, F., Lübbert, M. et al. Hemolytic crisis in a patient treated with eculizumab for paroxysmal nocturnal hemoglobinuria possibly triggered by SARS-CoV-2 (COVID-19): a case report. Ann Hematol (2020). https://doi.org/10.1007/s00277-020-04318-6

2. Risitano, A., Maciejewski, J., Muranski, P. et al. Large granular lymphocyte (LGL)-like clonal expansions in paroxysmal nocturnal hemoglobinuria (PNH) patients. Leukemia 19,217-222 (2005). https://doi.org/10.1038/sj.leu.2403617

3. Jia Yu, Xuan Yuan, Hang Chen, Shruti Chaturvedi, Evan M. Braunstein, Robert A. Brodsky; Direct activation of the alternative complement pathway by SARS-CoV-2 spike proteins is blocked by factor D inhibition. Blood 2020; 136 (18): 2080-2089. doi: https://doi.org/10.1182/blood.2020008248

4. Hrishikesh S. Kulkarni, John P. Atkinson; Targeting complement activation in COVID-19. Blood 2020; 136 (18): 2000-2001. doi: https://doi.org/10.1182/blood.2020008925

5. Risitano AM. Paroxysmal nocturnal hemoglobinuria and the complement system: recent insights and novel anticomplement strategies. Adv Exp Med Biol. 2013;735:155-72. doi: 10.1007/978-1-4614-41182_10. PMID: 23402025. 


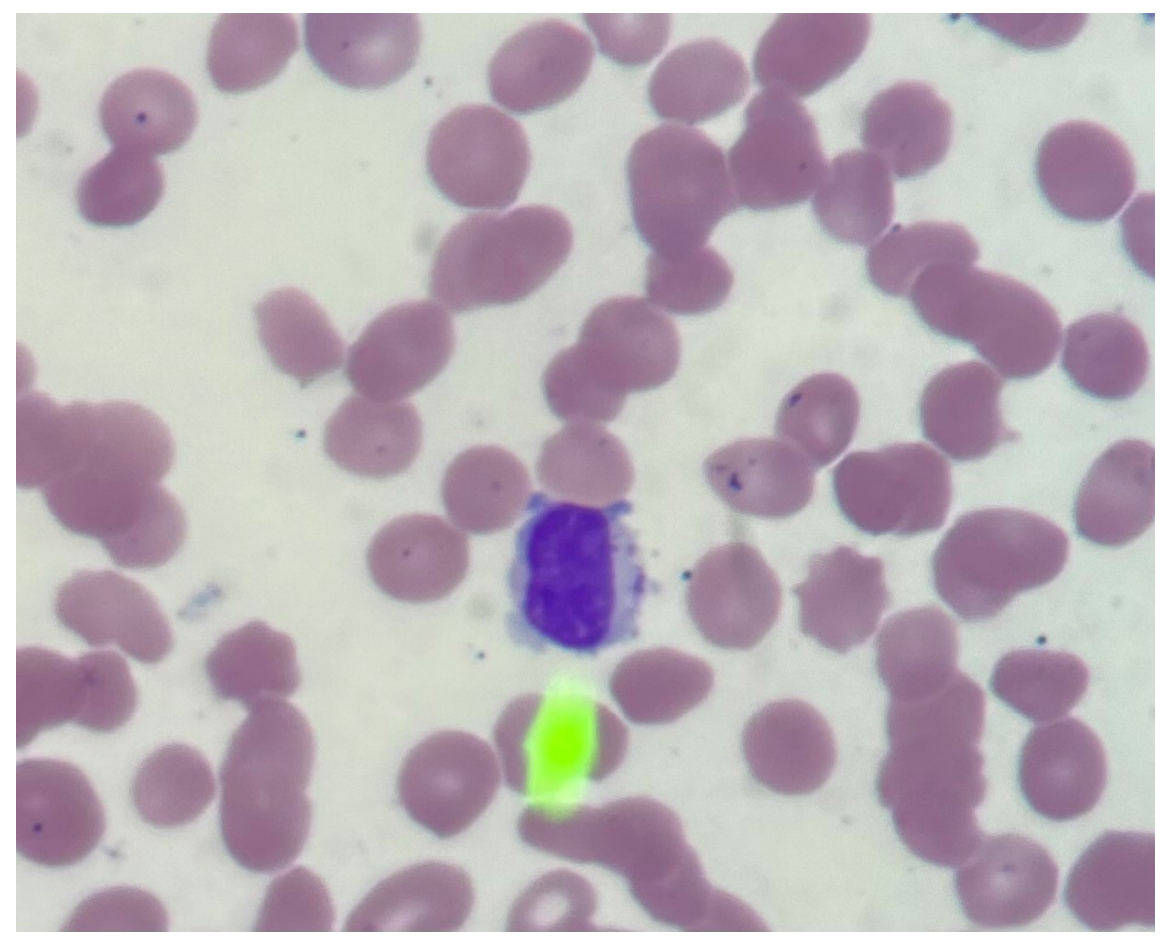

Test Performed: T Cell Gene Rearrangement

RESULTS:

TCR-beta: POSITIVE

TCR-gamma: POSITIVE

INTERPRETATION :

Discrete bands were identified in both TCR-beta and TCR-gamma analysis, suggesting a clonal T-cell population.

Results should always be interpreted with appropriate clinical and pathological data. Methods: Highly purified DNA was subjected to multiplex PCR using primers to conserved regions within the TCRB and TCRG genes. The limit of detection is 28.

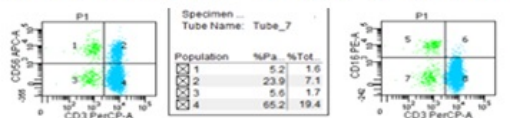

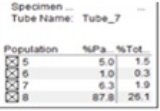

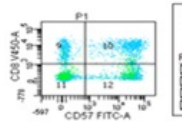

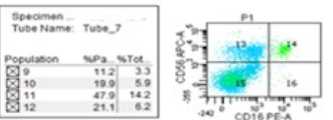

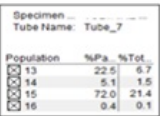

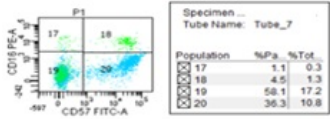

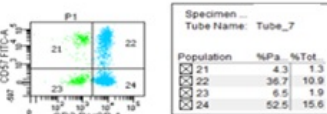

|

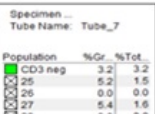

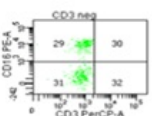

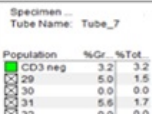




\begin{tabular}{|c|c|c|c|c|}
\hline \multicolumn{5}{|l|}{ RESULTS } \\
\hline Marker Name & Result & Unit & $H / L$ & Normal Range \\
\hline PNH RBC-Partial Ag Loss & 0.21 & $\%$ & & $0.00-0.99$ \\
\hline PNH RBC-Complete Ag Loss & 4.34 & $\%$ & $\mathrm{H}$ & $0.00-0.01$ \\
\hline PNH Granulocytes & 42.55 & $\%$ & $\mathrm{H}$ & $0.00-0.01$ \\
\hline PNH Monocytes & 45.84 & $\%$ & $\mathrm{H}$ & $0.00-0.05$ \\
\hline \multicolumn{5}{|l|}{ FINAL DIAGNOSIS } \\
\hline \multicolumn{5}{|c|}{ A population of RBCs, granulocytes, and monocytes with PNH phenotype is detected. } \\
\hline \multicolumn{5}{|c|}{$\begin{array}{l}\text { This finding is consistent with the diagnosis of PNH. Clinical correlation is recommended. Recent transfusion can } \\
\text { decrease the sensitivity of this test and interfere with accuracy. }\end{array}$} \\
\hline \multicolumn{5}{|c|}{$\begin{array}{l}\text { Granulocytes and monocytes with PNH phenotype are usually present in similar percentages in classical PNH cases. } \\
\text { RBCs with PNH phenotype are often present in a smaller percentage due to hemolysis and/or blood transfusion. In } \\
\text { cases of significant discrepancy repeat testing and clinical correlation are recommended. }\end{array}$} \\
\hline \multicolumn{5}{|c|}{ METHOD } \\
\hline \multicolumn{5}{|c|}{$\begin{array}{l}\text { Antibodies to the following antigens were used for cell gating and interpretation. RBCs: CD235a and CD59. WBCs: } \\
\text { CD14, CD15, CD 16, CD24, CD } 33, \text { CD } 45 \text {, FLAER. }\end{array}$} \\
\hline
\end{tabular}

